\title{
Experimental Demonstration of a Statistical OFDM-PON with Multiband ONUs and Elastic Bandwidth Allocation
}

\author{
Iván N. Cano, Xavier Escayola, Philipp C. Schindler, María C. Santos, Victor Polo, Juerg \\ Leuthold, Ioannis Tomkos, and Josep Prat
}

\begin{abstract}
The statistical OFDM-PON concept with multiband Optical Network Units (ONUs) is experimentally tested with two users and an Optical Line Terminal (OLT) at $2.5 / 5 \mathrm{~Gb} / \mathrm{s}$ total effective capacity with BPSK/QPSK modulation. Both downstream (DS) and uplink (UL) were measured based on intensity modulation and direct-detection (IMDD). The ONUs consisted of local non-preselected wavelength distributed feedback (DFB) laser sources centrally controlled to reduce overlapping probability. In addition, a radiofrequency (RF) mixing stage in the ONUs up/down-converts the user data to/from the OFDM signal reducing the computational effort. Compared with ONUs processing the whole signal, the multiband approach presents comparable results with almost symmetrical power budgets of around $25 \mathrm{~dB}$ and $20 \mathrm{~dB}$ with BPSK and QPSK respectively, which could increase up to $4.5 \mathrm{~dB}$ by allocating a spectral guard interval between the optical carrier and the OFDM data. Furthermore, elastic bandwidth allocation is explored which is shown to compensate for up to $18 \mathrm{~dB}$ differential link-loss.
\end{abstract}

Index Terms-Passive optical networks; PON; Orthogonal Frequency Division Multiplexing; OFDMAPON; statistical-PON; elastic bandwidth allocation; Intensity modulation direct-detection

\section{INTRODUCTION}

Orthogonal frequency division multiplexing access

Manuscript received July 1, 2014.

Iván N. Cano, Xavier Escayola, María C. Santos, Victor Polo and Josep Prat are with Universitat Politecnica de Catalunya (UPC), Jordi Girona 1-3, E-08034, Barcelona, Spain (e-mail: ivan.cano@ tsc.upc.edu).

Philipp C. Schindler was with Institute of Photonics and Quantum Electronics, Karlsruhe Institute of Technology, 76131, Karlsruhe, Germany and now is with Infinera Corp, Sunnyvale, CA, USA.

Juerg Leuthold was with Institute of Photonics and Quantum Electronics, Karlsruhe Institute of Technology, 76131, Karlsruhe, Germany and now is with Laboratory for Electromagnetic Fields and Microwave Electronics (IFH), Swiss Federal Institute of Technology Zurich (ETHZ).

Ioannis Tomkos is with Athens Information Technology Center (AIT), 19.5km Markopoulo Ave., Peania 19002, Athens, Greece.
(OFDMA) has attracted interest for research in passive optical networks (PON) due to features like fine bandwidth (BW) granularity and elastic BW provisioning [1-4]. Several studies have shown its feasibility for downstream (DS) though few have been related to the uplink (UL) multipointto-point. In $[5,6]$, an UL with centrally distributed optical carrier and coherent detection was demonstrated. As an alternative to reduce the cost of the optical network units (ONU) a statistical network with direct-modulated nonpreselected light sources was proposed [7-9]. In [7], the concept was studied in an OFDM-PON through statistical analysis and numerical simulation. It included a centralized thermal tuning control of random wavelength ONU lasers to reduce overlapping probability and limit interference.

In current PON deployments, each ONU processes the complete signal BW even if the user employs only a fraction of it. This overcapacity in the ONU has an effect on the power consumption [10]. In order to reduce the ONU complexity and lower its energy requirements, a multiband OFDM approach can be employed. In this technique, the ONUs include tunable radio-frequency (RF) oscillators to place/retrieve the assigned subcarriers (SC) to/from the multiuser OFDM signal [11, 12].

A system requirement demanded by operators for future PON is to reuse legacy network infrastructure. Hence, the differential link-loss among the users spread geographically from the central office becomes a major challenge [13]. In OFDM-PON, this concern can be addressed through dynamic assignment of SC and modulation levels to the users according to their power budget availability [14].

This paper experimentally validates a statistical multiband OFDM PON with two ONUs in both DS and UL including flexible BW allocation. It extends the results presented in [12] where only the UL was studied. It also compares the performance between the system in baseband and with a spectral guard interval (GI) between the optical carrier and the OFDM data signal. The technique reduces the processing effort in the ONU by setting their allocated BW into non-overlapping orthogonal frequency bands by means of a RF mixing stage. As a result, the fast Fourier transform (FFT) size in the ONU is decreased. Proper detection of data to/from both ONUs is achieved in DS/UL with performance values in line with those measured with digital SC allocation. In addition, the flexible BW allocation is also studied to compensate the difference in losses 
experienced by the users in the PON. The paper is organized as follows: a description of the network architecture with the statistical PON concept is presented first; the experimental setup is then described followed by the results and the conclusions.

\section{NeTWORK ARCHITECTURE: The STATISTICAL OFDM-PON CONCEPT}

A power splitter based PON tree architecture with service spectrum breakdown such as the Accordance network proposal [6] is considered (Fig. 1). Both the optical line terminal (OLT) and ONUs are based on intensitymodulation with direct-detection (IMDD) technique whose simplicity and performance is attractive for access networks [15-17]. In order to avoid backscattering effects, DS and UL are assigned to L-band and C-band respectively.

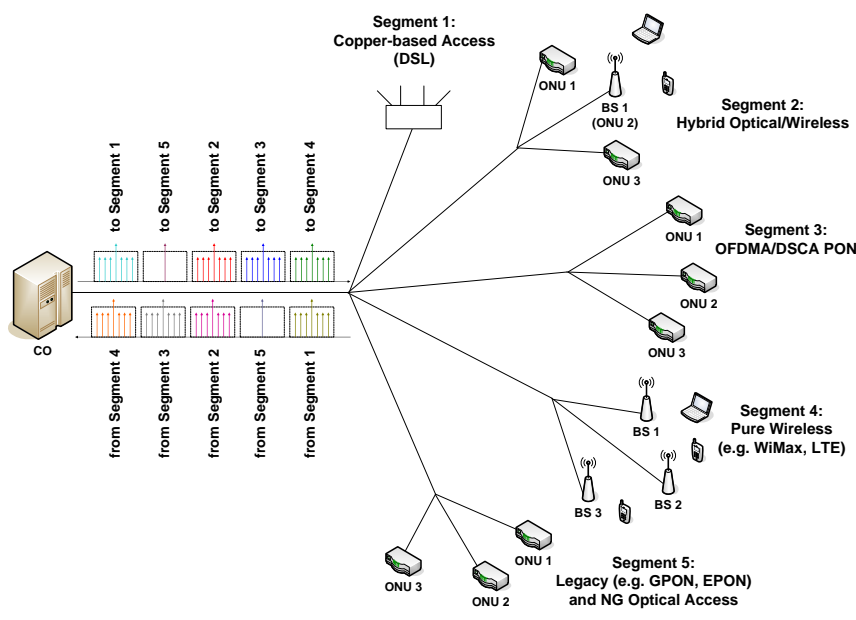

Fig. 1. Network architecture.

The ONU transmitter ( $\mathrm{Tx}$ ) consists of distributed feedback lasers (DFB) with non-preselected wavelength in the C-band. The use of such light sources would evade large inventory and complex provisioning to the operator. To avoid interference, the emission frequency of the ONU laser is regulated through temperature and current control circuits which allows for $\lambda$ shifts up to $1.6 \mathrm{~nm}$ with $0.1 \mathrm{~nm}$ steps. The tuning transition time is reduced to $2 \mu \mathrm{s}$ approximately by employing current transients and temperature changes simultaneously [18].

The OLT includes an intelligent activation and operation algorithm like those proposed in $[19,20]$ to initialize and track the $\lambda$ of the ONUs lasers by centrally measuring and sending instructions to the control circuits. As a result, the ONU rejection probability is minimized [7]. The ONUs $\lambda$ are kept at enough spectral distance $(0.1 \mathrm{~nm})$ to reduce the optical beat interference (OBI) at the OLT below a quality threshold. Since the ONU lasers emit at random wavelengths and are centrally managed to maintain the overall performance, it is referred as statistical PON.

\section{EXPERIMENTAL SETUP}

The setup contained an OLT and two ONUs emulating a PON as depicted in Fig. 2. For the OLT Tx a total of $2^{19}$ bits were randomly generated and mapped to either binary phase-shift keying (BPSK) or quadrature-PSK (QPSK). The symbols were arranged to have Hermitian symmetry in a 256-point inverse Fast Fourier Transform (iFFT) to get a real valued OFDM signal. This FFT size was chosen because it is currently achievable in FPGA real-time processing [21]. No cyclic prefix was added since for the fiber lengths considered no significant chromatic dispersion was generated. The first half of the BW carried the DS information of $\mathrm{ONU}_{1}$, whereas the second were filled with $\mathrm{ONU}_{2}$ DS data. The OFDM signal was loaded to an arbitrary waveform generator (AWG) which produced samples at $5 \mathrm{GSa} / \mathrm{s}$ with 8-bits resolution digital-to-analog converters (DAC). This entailed a maximum effective capacity of $1.25 \mathrm{~Gb} / \mathrm{s}$ for each user with BPSK modulation and of $2.5 \mathrm{~Gb} / \mathrm{s}$ with QPSK. The electrical output was amplified and modulated a DFB emitting at $1600.85 \mathrm{~nm}$ through a Mach-Zehnder modulator (MZM) biased at quadrature with an insertion loss of almost $6 \mathrm{~dB}$. The optical output power was limited to $0 \mathrm{dBm}$. The optical signal was then sent to the optical distribution network (ODN) after a

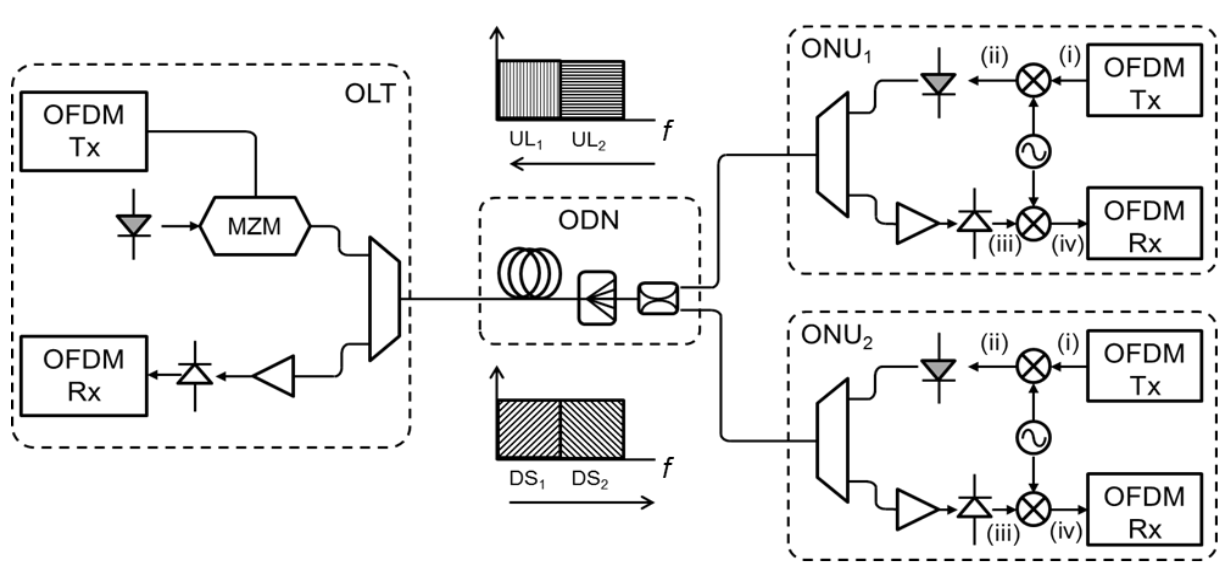

(a) (i)

(ii)

(iii)

(iv)

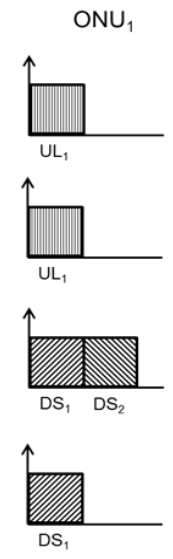

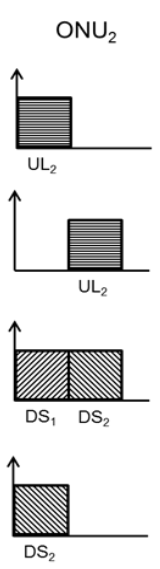

(b)

Fig. 2. (a) Experimental setup block diagram; (b) Representation of the data spectra in $\mathrm{ONU}_{1}$ and $\mathrm{ONU}_{2}$. 
$\mathrm{red} / \mathrm{blue}$ filter.

The OLT Rx involved a single $10 \mathrm{GHz}$ photodiode (PD) preceded by an erbium doped fiber optical amplifier (EDFA) which kept the input optical power to the PD at $-9 \mathrm{dBm}$. The signal was then sampled with a 50GSa real time oscilloscope (RTO). The digital samples were demodulated with a 256-point FFT, followed by a 1-tap equalizer whose coefficients were set by a known training sequence in the initial four OFDM symbols and the bit error ratio (BER) for each ONU UL signal was computed.

The ODN entailed $25 \mathrm{~km}$ of single mode fiber, an optical attenuator of $12 \mathrm{~dB}$ and a $3 \mathrm{~dB}$ optical coupler. The total loss of the ODN was $20 \mathrm{~dB}$. In Fig. 2 a representation of the transmitted spectra in the PON is shown.

At the ONUs, the Tx generated $2^{18}$ random bits which were mapped to either BPSK or QPSK, accommodated for Hermitian symmetry and modulated with a 128-point iFFT each. The UL samples of each ONU were loaded separately to two channels of an AWG with 8-bit resolution. Each user signal had $2.5 \mathrm{GHz}$ BW. The resulting baseband signals were shifted to their corresponding frequency slot through an RF mixer (Fig. 2b) and then directly modulated a DFB laser with $5 \mathrm{GHz} \mathrm{BW}$. The output power of each ONU was set to $0 \mathrm{dBm}$. The optical $\mathrm{Tx}$ signal passed through a $\mathrm{red} / \mathrm{blue}$ filter and it was joined in a $3 \mathrm{~dB}$ optical coupler with the contribution from the other ONU. The total UL capacity was thus equal to that of the DS signal. Then, the composite OFDM UL signal was launched into the ODN towards the OLT where it was detected. As for the ONU Rx, after the red/blue filter, the isolated DS component was detected by a $\mathrm{p}-\mathrm{i}-\mathrm{n} \mathrm{PD}$ preamplified by a semiconductor optical amplifier (SOA) with $18 \mathrm{~dB}$ gain and noise figure (NF) of 6.5dB. Alternatively, for simplifying the Rx, we also tested an avalanche PD (APD). An RF down-conversion stage then took the DS spectrum corresponding to the ONU down to baseband. The electrical signal was low-pass filtered and sampled with a 50GSa RTO. The samples were demodulated with a 128-points FFT, equalized, demapped and the BER was computed.

\section{RESULTS}

We first emulated the activation process in the statistical PON with efficient optical BW occupation. We tuned $\mathrm{ONU}_{2}$ $\lambda$ to transmit as close as possible to $\mathrm{ONU}_{1}$ while limiting the OBI below a minimum value [22-24]. The original and final wavelengths are shown in Fig. 3.
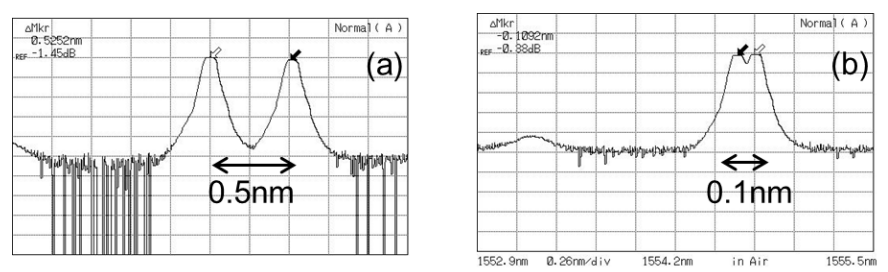

Fig. 3. (a) Initial and (b) final $\lambda$ separation between ONUs.

Initially, $\mathrm{ONU}_{1}$ and $\mathrm{ONU}_{2}$ emitted at $1554.7 \mathrm{~nm}$ and $1554.2 \mathrm{~nm}$ respectively. $\mathrm{ONU}_{1}$ was first connected to the PON and afterwards $\mathrm{ONU}_{2}$ was turned on (Fig. 3a). As measured in [7, 23], a spectral separation of $0.1 \mathrm{~nm}$ between users was enough to avoid penalties. To use optical BW more efficiently, $\mathrm{ONU}_{2}$ was tuned to displace its emission $\lambda$ rapidly by $0.4 \mathrm{~nm}$ to $1554.6 \mathrm{~nm}$ (Fig. $3 \mathrm{~b}$ ) where the reception quality was maintained [7, 23].

\section{A. Downstream}

The DS signal was generated with a single FFT at the OLT. The lower and higher half of the spectrum were reserved for $\mathrm{ONU}_{1}$ and $\mathrm{ONU}_{2}$ respectively. Each $\mathrm{ONU}$ then detected the complete optical signal but processed only its corresponding SC.

As a first test, both ONUs equally shared the available $\mathrm{SC}$. The frequency of the RF mixer in $\mathrm{ONU}_{2}\left(\mathrm{f}_{\mathrm{IF} 2}\right)$ was set to $1.25 \mathrm{GHz}$. ONU 1 did not need any $\mathrm{RF}$ stage as it was assigned the lowest spectral band in the OFDM signal, which started at baseband. Then each ONU only processed their own half of the SC. Fig. 4 plots the BER against the Rx power for both ONUs with BPSK and QPSK modulation. At a forward error correction (FEC) target of $\mathrm{BER}=10^{-3}$, both ONUs detected at approximately $-25 \mathrm{dBm}$ and $-20 \mathrm{dBm}$ the BPSK and QPSK signal respectively. Considering that $\mathrm{P}_{\mathrm{Tx}}=0 \mathrm{dBm}$, the power budget available was $20 \mathrm{~dB}$ and $25 \mathrm{~dB}$ for QPSK and BSPK correspondingly. The difference in the $\mathrm{Rx}$ power between the modulation formats could give the possibility to switch them according to the power budget available and BW demand of each user. The small difference observed in the Rx sensitivities among the ONUs was mainly caused by small drifts in $\mathrm{ONU}_{2} \mathrm{RF}$ oscillator.

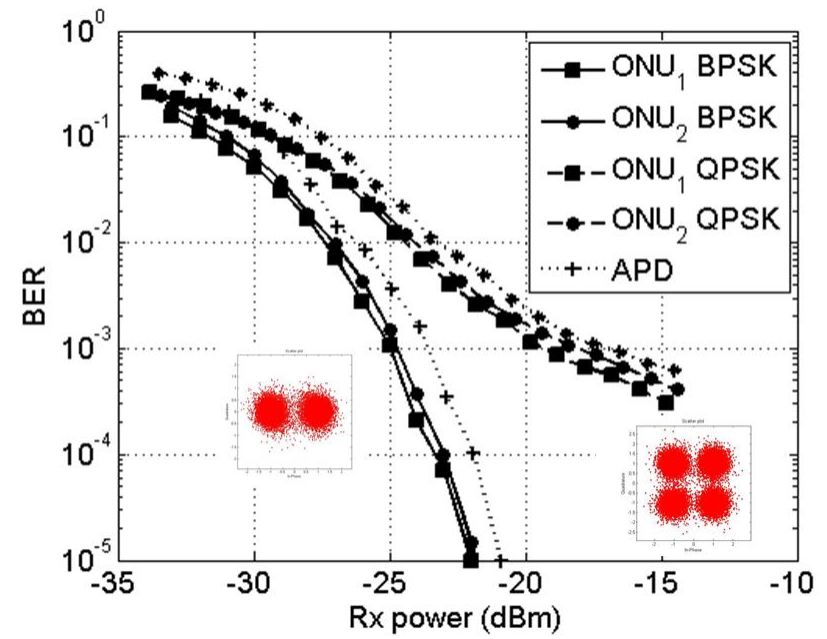

Fig. 4. BER against Rx power for the two ONUs equally sharing the total BW with BPSK and QPSK modulation in DS. The dotted lines correspond to the averaged curves of both ONUs with APDbased $\mathrm{Rx}$. The constellations are for $\mathrm{BER}=10^{-3}$ in $\mathrm{ONU}_{1}$.

Since the ONU is a cost-sensitive element in the PON, we replace the preamplified $\mathrm{PD}$ in $\mathrm{Rx}$ with an APD (FRM5N143DS). Fig. 4 includes the averaged performance graphs over both ONUs when employing the APD with BPSK and QPSK. There is a penalty of about $1.5 \mathrm{~dB}$ for the APD Rx for both modulation formats. However, the power budget can be kept by increasing the launched power to $2 \mathrm{dBm}$ with the advantage of a simpler ONU Rx.

In a second set of experiments, a spectral bandgap (BG) with empty $\mathrm{SC}$ was considered between the data spectrum of $\mathrm{ONU}_{1}$ and $\mathrm{ONU}_{2}$. We left unchanged $\mathrm{f}_{\mathrm{IF} 2}$ and discarded 
the unused SC in each ONU after demodulation. The effective $\mathrm{BW}$ per ONU (BWONU), measured as the operative SC by the total available, was thus, reduced. The $\mathrm{Rx}$ sensitivity at $\mathrm{BER}=10^{-3}$ against $\mathrm{BW}$ ONU for both ONUs is shown in Fig. 5 with BPSK and QPSK modulation.

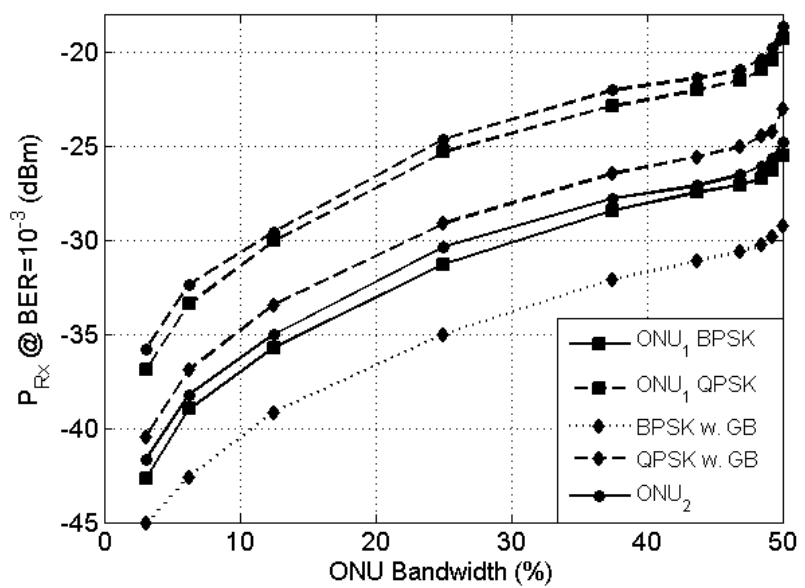

Fig. 5. Rx sensitivity at $\mathrm{BER}=10^{-3}$ against effective data $\mathrm{BW}$ for both ONUs with BPSK and QPSK modulation formats with multiband OFDM. The dotted lines correspond to $\mathrm{ONU}_{1}$ curves with a spectral GI.

We observed that as the BWonu decreased, the $\mathrm{Rx}$ sensitivity improved. A value of $P_{R x}=-32 \mathrm{dBm}$ was reached when each ONU employed only $25 \%$ of the BW with BPSK. Furthermore, QPSK could be detected with only around $4.5 \mathrm{~dB}$ penalty as compared with BSPK. We also noticed from Fig. 5 that $\mathrm{ONU}_{2}$ had almost $1 \mathrm{~dB}$ penalty with respect to $\mathrm{ONU}_{1}$ which was attributed to small drifts in the electrical oscillator frequency whose tolerance was limited to $\pm 5 \mathrm{kHz}$ [14].

When the OFDM signal is direct-detected, there are mixing terms between the SC that appear in the data frequency band degrading the performance. A spectral GI between the optical carrier and OFDM signal equal to the signal BW is typically used to keep the mixing terms out of the data band [25]. In our setup, the maximum oscillator frequency of the $\mathrm{RF}$ stages is $6 \mathrm{GHz}$. This entails a maximum GI of $3.5 \mathrm{GHz}$ in the DS signal. An RF stage is added in the OLT and $\mathrm{ONU}_{1} \mathrm{f}_{\mathrm{IF} 1}$ is also tuned to $6 \mathrm{GHz}$. $\mathrm{ONU}_{2}$ is not used because $\mathrm{f}_{\mathrm{IF} 2}>6 \mathrm{GHz}$ is required. Fig. 5 includes the Rx sensitivity curve of $\mathrm{ONU}_{1}$ with GI. There is an improvement of nearly $4.5 \mathrm{~dB}$ for both BPSK and QPSK. However, this enhancement comes at the expense of a wider spectrum in the OFDM signal,

For comparison, we carried out the same experiment with ONUs processing the complete DS signal, i.e. with a $256^{-}$ point FFT. For the BER computation each ONU considered only its corresponding half of SC, and the rest were discarded. The obtained results (plotted in Fig. 6) were comparable with those in Fig. 5 for multiband OFDM. The latter presented a penalty for BWONU $>45 \%$ due to the nonideal low-pass filter response after the RF stage in $\mathrm{Rx}$.

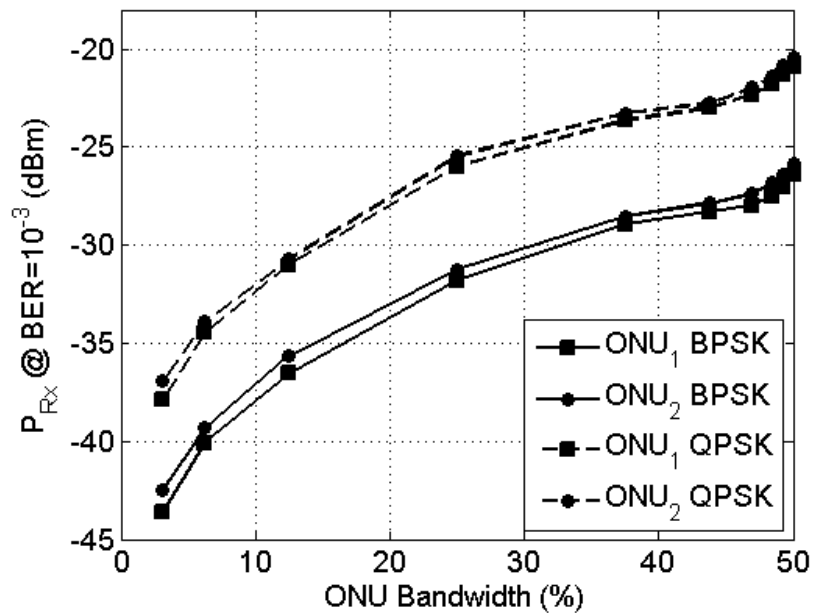

Fig. 6. Rx sensitivity at $\mathrm{BER}=10^{-3}$ against effective data $\mathrm{BW}$ for both ONUs with BPSK and QPSK modulation formats when the ONUs process the whole BW.

\section{B. Uplink}

For the UL performance assessment, the multiband OFDM contributions of both ONUs were detected with a single 256-point FFT in the OLT. In order to reuse the electrical oscillator, the SC assignment followed the same scheme as in DS. Since the ONUs UL spectra had to be accurately synchronized, we carefully set identical electrical and optical paths between the $3 \mathrm{~dB}$ optical coupler and the ONUs electrical Tx generator. In a commercial network, the ONUs could be coordinated through the ranging protocol during the activation process. Fig. 7 plots the BER against the Rx sensitivity computed at the OLT for both ONUs with BPSK and QPSK modulation.

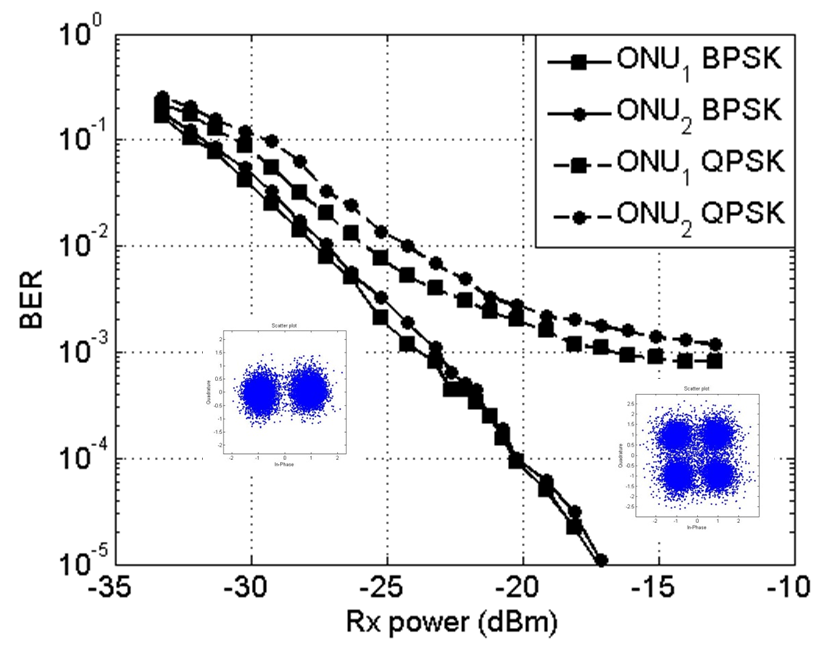

Fig. 7. BER against $\mathrm{Rx}$ power for both ONUs with BPSK and QPSK modulation. The constellations are for $\mathrm{ONU}_{1}$ at $\mathrm{BER}=10^{-3}$.

Both ONUs were detected properly below the target $\mathrm{BER}=10^{-3}$ at $-24 \mathrm{dBm}$ and $-18 \mathrm{dBm}$ for BPSK and QPSK respectively. However, there was a BER floor appearing in the QPSK signal close to $\mathrm{BER}=10^{-3}$. The same was observed for the BPSK signal but the floor was below $B E R=10^{-5}$. This motivated to look for the errors in each SC. Fig. 8 shows the number of errors against the SC number for both ONUs. Interestingly, the errors occurred in the SC closest to the 
other ONU part caused by a slight overlapping due to the spectrum tails, as seen in the inset of Fig. 8.

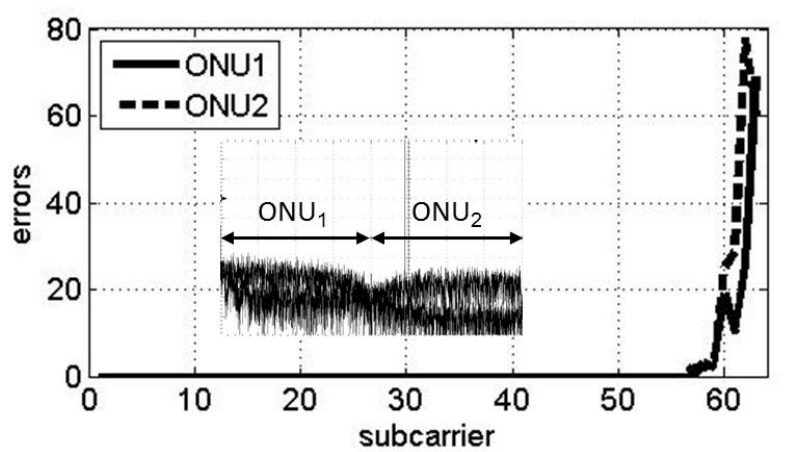

Fig. 8. Total errors against SC number.

In order to improve the performance, a spectral BG was set between the ONU signals. Each ONU occupied $25 \%$ of the BW and both were spectrally together firstly. Then $\mathrm{ONU}_{2}$ spectrum was separated from $\mathrm{ONU}_{1}$ by tuning $\mathrm{f}_{\mathrm{IF} 2}$. The OLT processed both the ONUs data spectra and the BG. The Rx sensitivity at $\mathrm{BER}=10^{-3}$ for both ONUs is plotted against the spectral BG in Fig. 9. For a $3 \mathrm{~dB}$ penalty, a $6.25 \%$ frequency $\mathrm{BG}$, i.e. $312 \mathrm{MHz}$, is needed for QPSK. This value is reduced to a moderate $1.56 \%(78 \mathrm{MHz})$ for BPSK because of its lower signal to noise ratio (SNR) requirement.

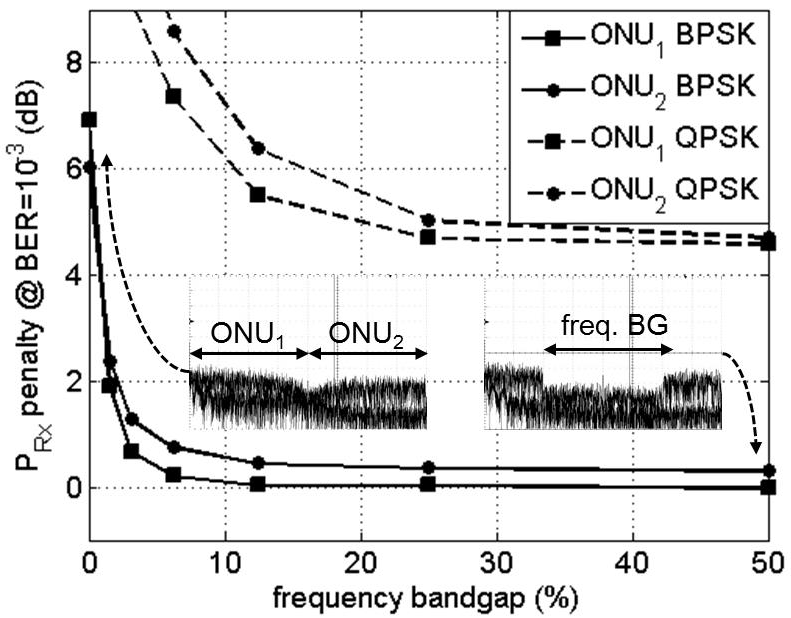

Fig. 9. Rx power for $\mathrm{BER}=10^{-3}$ against frequency $\mathrm{BG}$. The insets illustrate the increase of the frequency BG between the ONU contributions.

The elastic BW allocation was also evaluated in UL. The BW occupied by each of the ONUs was lowered by zeropadding (ZP) some of the SC. As a result, the spectral separation between the users was also increased improving the $\mathrm{Rx}$ sensitivity. Fig. 10 shows the $\mathrm{Rx}$ optical power needed at BER $=10^{-3}$ for both ONUs against BWonU with BPSK and QPSK modulation. As expected from Fig. 9 results, there were penalties for $\mathrm{BWONU}_{\mathrm{ON}}>45 \%$ due to spectral overlapping in the neighboring user SC (insets of Fig. 10). Notably, the curves are similar to the ones obtained for the DS case. Due to the limited BW of the DFB lasers, a spectral GI was not tested in the UL. However, a performance improvement is expected if a spectral GI is left between the OFDM signals and the optical carriers provided that the frequency separation between the users is correspondingly increased to prevent OBI.

When increasing the $\mathrm{ZP}$, besides enlarging the spectral $\mathrm{BG}$, the total power is distributed into fewer SC, increasing their SNR. Hence, clearer constellations and improved Rx sensitivity are obtained yet at the price of a lower effective bitrate. The Rx sensitivity when reducing BWonu from $50 \%$ to $25 \%$ improves by $6 \mathrm{~dB}$ for BPSK. Similar values are obtained for DS (Fig. 5). For QPSK, the Rx sensitivity is as low as $-24.5 \mathrm{dBm}$ with $\mathrm{BW}_{\mathrm{ONU}}=25 \%$, almost $8 \mathrm{~dB}$ better than with $\mathrm{BW}$ ONU $=50 \%$. The performance is slightly better for $\mathrm{ONU}_{1}$ which operated in baseband than for $\mathrm{ONU}_{2}$ because of small drifts in $\mathrm{f}_{\mathrm{IF} 2}$ and the non-ideal laser response which caused some distortion in the higher part of the spectrum.
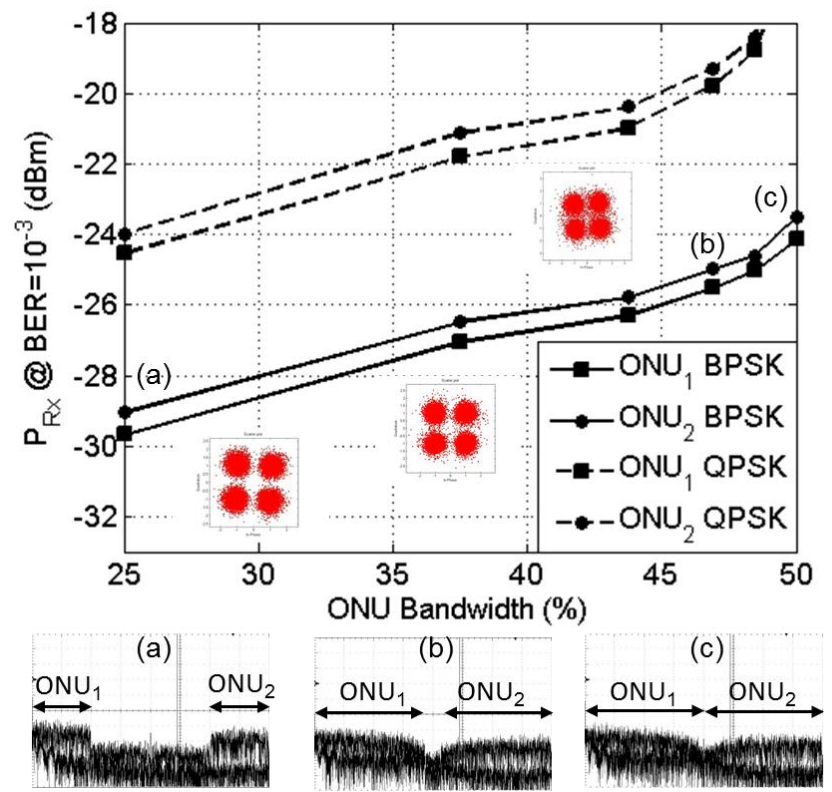

Fig. 10. Rx sensitivity at $\mathrm{BER}=10^{-3}$ against $\mathrm{BW}$ ONU for both ONUs with BSPK and QPSK modulation in multiband OFDM. The insets show the spectrum of each ONU contribution in the Rx signal.

We also tested the UL with ONUs processing the complete signal with BPSK, QPSK, and several ZP percentages. The Rx sensitivity against BWonu measured at the OLT for each ONU is plotted in Fig. 11. The results were around $1 \mathrm{~dB}$ better than the multiband OFDM due to the frequency drifts in the RF mixer. For BWonU $>45 \%$ the non-ideal electrical filters used in multiband OFDM to confine and limit the BW of each ONU did not remove the spectral tails completely producing overlapping when the UL contributions were combined. Thus, in these cases the Rx sensitivity of the ONUs processing the whole OFDM signal, improved by $2 \mathrm{~dB}$. However, the multiband OFDM has the relevant advantage of lowering the ONU complexity by reducing the FFT size through the help of an RF mixer. This translates directly into the power consumption which is also lowered. 


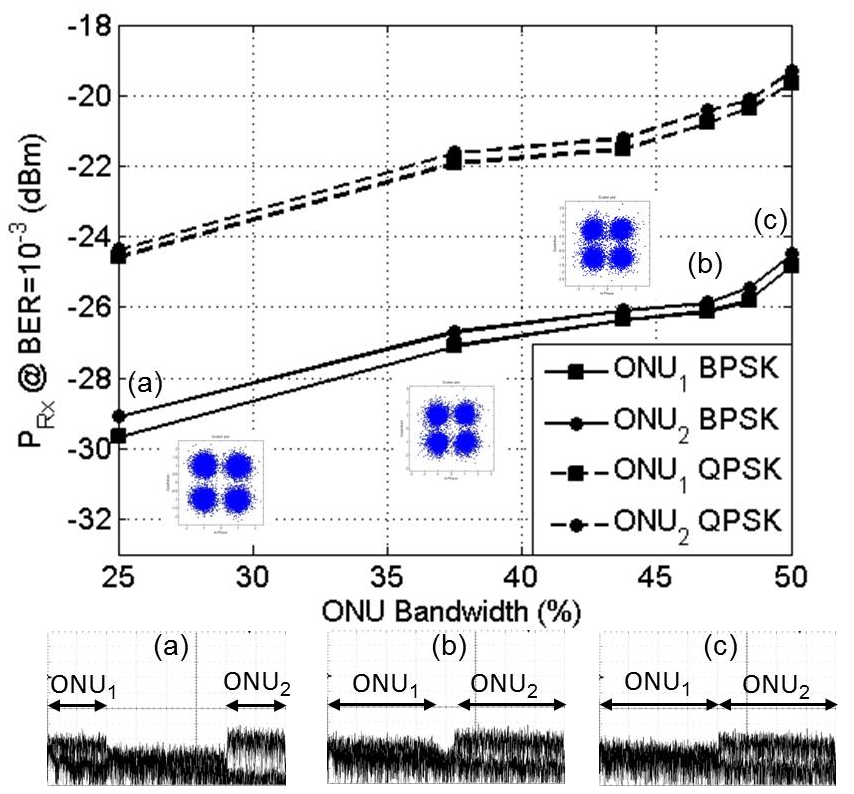

Fig. 11. Rx sensitivity at $\mathrm{BER}=10^{-3}$ against effective data $\mathrm{BW}$ for both ONUs with BSPK and QPSK modulation with each ONU processing the whole BW. The insets show the spectrum of each ONU contribution in the detected signal.

To further evaluate the relation between the $\mathrm{Rx}$ sensitivity, modulation format and active SC, the experiment was extended to 8-PSK and 16-QAM. Fig. 12 summarizes the $\mathrm{Rx}$ sensitivity needed at $\mathrm{BER}=10^{-3}$ (level lines) as a function of both the modulation format and BWONU considering an average over the two ONUs.

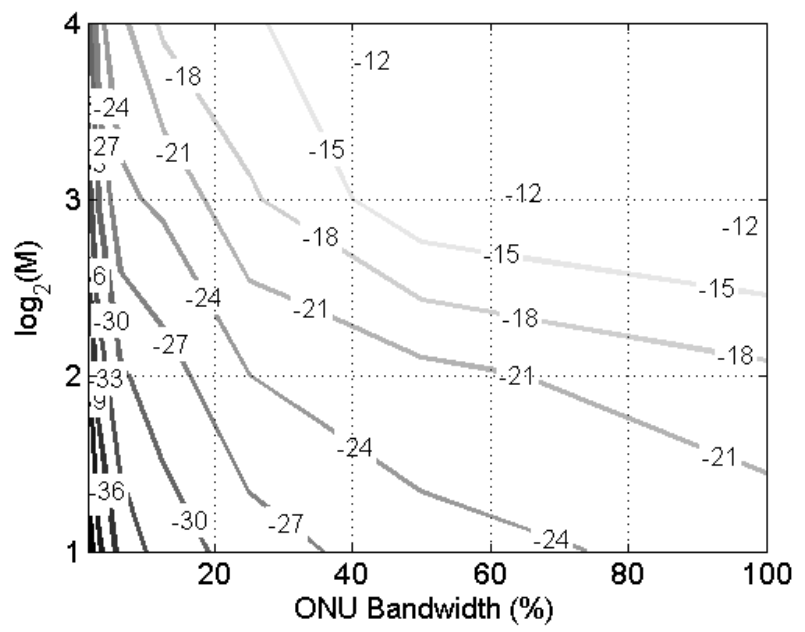

Fig. 12. Rx sensitivity at $\mathrm{BER}=10^{-3}$ level lines (in $\mathrm{dBm}$ ) as a function of modulation format and BWONU. (M: modulation bits).

The lines in Fig. 12 can be used to allocate the active SC and modulation format for each ONU according to the users BW need and available power budget. The negotiation can be carried out at the registration process or dynamically during operation. As an example, for the ODN shown in Fig. 2 , if $\mathrm{ONU}_{1}$ transmits and receives a QPSK signal with BWONU $=3.12 \%\left(\mathrm{P}_{\mathrm{Rx}}=-33 \mathrm{dBm}\right)$ and $\mathrm{ONU}_{2}$ uses BPSK with $B W_{O N U}=96.8 \%\left(P_{R x}=-23 \mathrm{dBm}\right)$, there is a difference of $10 \mathrm{~dB}$ in the $\mathrm{Rx}$ power needed. This value translates into a fiber length separation of $50 \mathrm{~km}$ or an extra splitting ratio of $1: 8$.
Therefore, the differential link-loss can be compensated and both users are detected properly.

As a final test, the SC allocation was assessed experimentally in UL by transmitting a short video signal. The complete description and results can be seen in [26].

\section{Conclusion}

A statistical OFDMA PON with multiband ONUs based on simple independent non-preselected DFB lasers was experimentally evaluated. The assessment included both DS and UL in separate optical bands with almost symmetrical Rx sensitivities of $-25 \mathrm{dBm}$ at $\mathrm{BER}=10^{-3}$ with BPSK. A penalty of $5 \mathrm{~dB}$ was obtained when the modulation format was extended to QPSK. The total effective capacity was $2.5 / 5 \mathrm{~Gb} / \mathrm{s}$ for BPSK and QPSK respectively. The digital SC allocation was also tested and the Rx sensitivity improved up to $2 \mathrm{~dB}$ when compared with the multiband technique. However, the advantage of the latter was that it allowed the ONU to process only its intended BW. The technique placed the ONU BW in its corresponding spectral band with the aid of an RF mixer, thus reducing the complexity and energy consumption. For the UL, a spectral BG of $6.25 \%$ was required to detect simultaneously both ONUs at the OLT with QPSK while keeping the penalty below $5 \mathrm{~dB}$ with respect to BPSK. The flexibility of OFDM to grant different SC and modulation formats independently to ONUs was also studied. The Rx sensitivity was enhanced by almost $6 \mathrm{~dB}$ when BWonu was reduced from $50 \%$ to $25 \%$. The results showed that the statistical OFDM PON could adapt to the users BW requirement and power budget availability. As a result, the $\mathrm{BW}$ can be allocated intelligently and dynamically through media access control protocols to compensate a differential link-loss of up to $18 \mathrm{~dB}$ among the users in the PON.

\section{ACKNOWLEDGMENT}

This work was supported in part by the European FP-7 projects ACCORDANCE and COCONUT, the Spanish Ministry of Science and Innovation under Grant TEC201125215 (ROMULA) and Mexican Science and Technology Council (CONACYT) under grant 185291.

\section{REFERENCES}

[1] D. Qian, N. Cvijetic, J. Hu, and T. Wang, "A novel OFDMAPON architecture with source-free ONUs for next-generation optical access networks," IEEE Photon. Technol. Let., vol.21, pp. 1265-1267, Sept. 2009.

[2] N. Cvijetic, "OFDM for next-generation optical access networks," IEEE J. Lightwave Technol., vol. 30, pp.384-398, Feb. 2012.

[3] K. Kanonakis, I. Tomkos, H. G. Krimmel, F. Schaich, C. Lange, E. Weis, J. Leuthold, M. Winter, S. Romero, P. Kourtessis, M. Milosavljevic, I. N. Cano, and J. Prat, "An OFDMA-based optical access network architecture exhibiting ultra-high capacity and wireline-wireless convergence," IEEE Commun. Mag., vol. 50, pp. 71-78, Aug. 2012.

[4] W. Lim, P. Kourtesis, M. Milosavljevic, and J. M. Senior, "Dynamic subcarrier allocation for 100Gbps, $40 \mathrm{~km}$ OFDMAPONs with SLA and CoS," IEEE J. Lightwave Technol., vol. 31, pp. 1055-1062, April 2013.

[5] J. von Hoyningen-Huene, H. Griesser, M. H. Eiselt, W. Rosenkranz, "Experimental demonstration of OFDMA-PON 
uplink-transmission with four individual ONUs," presented at OFC 2013, Anaheim CA, Mar. 19-21, paper OTh3A.2.

[6] C. Ruprecht, Y. Chen, D. Fritzsche, J. von Hoyningen-Huene, N. Hanik, E. Weis, D. Breuer, W. Rosenkranz, "37.5-km urban field trial of OFDMA-PON using colorless ONUs with dynamic bandwidth allocation and TCM," presented at OFC 2014, San Francisco, CA, Mar. 11-13, paper Th3G.5.

[7] I. Cano, M. C. Santos, V. Polo, and J. Prat, "Dimensioning of OFDMA PON with non-preselected independent ONUs sources and wavelength-control," Optics Express, vol. 20, pp. 607-613, Jan. 2012.

[8] W. Poehlmann and T. Pfeiffer, "Demonstration of wavelengthset division multiplexing for a cost effective PON with up to 80Gbit/s upstream bandwidth," presented at ECOC 2011, Geneva, Switzerland, Sept. 18-22, paper We.9.C.1.

[9] N. Cheng, G. Wei, and F. Effenberger, "Dynamic spectrum managed passive optical networks," IEEE Commn. Mag, vol. 49, pp. 86-93, Nov. 2011.

[10] P. Vetter, "Next generation optical access technologies," presented at ECOC 2012, Amsterdam, Netherlands, Sept. 1620, paper Tu.3.G.1.

[11] B. Charbonnier, A. Lebreton, P. Chanclou, G. Beninca, S. Mexezo, R. Dong, and J. LeMasson, "Low complexity FDM/FDMA approach for future PON," presented at OFC 2013, Anaheim, CA, Mar. 19-21, paper OTh3A.7.

[12] I. Cano, X. Escayola, P. Schindler, M. Santos, V. Polo, J. Leuthold, and J. Prat, "Experimental demonstration of multiband upstream in statistical OFDM-PONs and comparison with digital subcarrier assignment," presented at OFC 2014, San Francisco, CA, Mar. 11-13, paper Th3G.4.

[13] P. Chanclou, A. Cui, F. Geilhardt, H. Nakamura, and D. Nesset, "Network operator requirements for the next generation of optical access networks," IEEE Network, vol. 26, pp. 8-14, Mar. 2012.

[14] I.N. Cano, A. Peralta, V. Polo, X. Escayola, M. Santos, J. Prat, "Differential link-loss compensation through dynamic bandwidth assignment in statistical OFDMA-PON," presented at OFC 2013, Anaheim CA, Mar. 19-21, paper OTh3A.5

[15] A. Dochhan, H. Griesser, L. Nadal, M. H. Eiselt, M. Svaluto, J. P. Elbers, "Experimental investigation of discrete multitone transmission in the presence of optical noise and chromatic dispersion," presented at OFC 2014, San Francisco, CA, Mar. 11-13, paper Tu2G.7.

[16] S. L. Jansen, B. Spinnler, I. Morita, S. Randel, and H. Tanaka, "100GbE: QPSK versus OFDM," Optical Fiber Technology, vol. 15, pp. 407-413, Dec. 2009.

[17] J. Tang, E. Hugues-Salas, R. P. Giddings, "First experimental demonstration of real-time adaptive transmission of $20 \mathrm{~Gb} / \mathrm{s}$ dual-band optical OFDM signals over 500m OM2 MMFs," presented at OFC 2013, Anaheim, CA, Mar. 19-21, paper OTh3A.1.

[18] V. Polo, P. Borotau, A. Lerín, and J. Prat, "DFB laser reallocation by thermal wavelength control for statistical udWDM in PONs," accepted for presentation at ECOC 2014.

[19] D. van Veen, W. Pöhlmann, J. Galaro, B. Deppisch, A. Duque, M. F. Lau, B. Farah, T. Pfeiffer, and P. Vetter, "System demonstration of a time and wavelength-set division multiplexing PON," presented in ECOC 2013, London, UK, Sept. 22-26, paper We.3.F.2.

[20] S. Kaneko, T. Yoshida, S. Furusawa, M. Sarashina, H. Tamai, A. Suzuki, T. Mukojima, S. Kimura, N. Yoshimoto, "First $\lambda^{\text {- }}$ tunable dynamic load-balancing operation enhanced bt $3^{-}, \mathrm{sec}$ bidirectional hitless tuning on symmetric 40-Gbit/s WDM/TDM-PON," presented in OFC 2014, San Francisco, CA, Mar. 9-13, paper Th5A.4

[21] "VIrtex-6 family overview," Xilinx Inc. Jan. 2010.

[22] C. Desem, "Optical interference in subcarrier multiplexed systems with multiple optical carriers," IEEE J. Select. Areas Commun., vol. 8, pp. 1290-1295, Sept. 1990.

[23] X. Q. Jin and J. M. Tang, "Experimental investigations of Wavelength Spacing and Colorlessness of RSOA-Based ONUs in Real-Time Optical OFDMA PONs", IEEE J. Lightwave Technol., vol. 30, pp. 2603-2609, Aug. 2012.

[24] X. Q. Jin, J. Groenewald, E. Hughes-Salas, R. P. Giddings, and J. M. Tang, "Upstream power budgets of IMDD optical OFDMA PONs incorporating RSOA intensity modulator-based colorless ONUs," IEEE J. Lightwave Technol., vol. 31, pp. 1914-1920, June 2013.

[25] B. J. C. Schmidt, A. J. Lowery, and J. Armstrong, "Experimental demonstrations of electronic dispersion compensation for long-haul transmission using directdetection optical OFDM," IEEE J. Lightwave Technol., vol. 26, pp. 196-203, Jan. 2008.

[26] I. Cano, X. Escayola, P. Schindler, M. Santos, V. Polo, J. Leuthold, and J. Prat (2013, Dec. 6). Upstream statistical OFDM low-cost solution [Online]. Available: https://www.youtube.com/watch?v=0lbuYu1Cxkc 